\title{
Amigdalitis hemorrágica espontánea abscedada: Revisión bibliográfica y reporte de un caso
}

\section{Spontaneous tonsillar hemorrhage and abscess: Bibliographic review and case report}

\author{
Loreto Álvarez S1, Matías Gómez G²,
}

\begin{abstract}
RESUMEN
En este artículo se presenta un paciente que en contexto de un cuadro amigdalino agudo bilateral con un absceso periamigdalino unilateral concomitante presenta sangrado espontáneo proveniente de la amígdala abscedada. Los signos y síntomas observados en este paciente, así como los estudios complementarios son compatibles con la entidad clínica definida como amigdalitis hemorrágica espontánea, una complicación altamente infrecuente de la amigdalitis aguda y/o crónica en nuestra época, la que solía tener una gran tasa de mortalidad y gravedad en la era preantibiótica. A continuación, se reúnen y analizan los antecedentes descritos en la literatura referidos a este cuadro, al igual que su estudio complementario requerido para definir conducta, la cual es fundamentalmente de resorte quirúrgico.

Palabras clave: Amigdalitis hemorrágica espontánea abscedada, amigdalitis hemorrágica, amigdalitis bilateral, hemorragia amigdalina espontánea, amigdalectomía.
\end{abstract}

\begin{abstract}
In this article we report a patient who, in the context of a bilateral acute tonsillar condition with a concomitant unilateral peritonsillar abscess presents spontaneous bleeding from the abscessed tonsil. The clinical features observed in our patient, as well as the complementary studies are all compatible with the clinical entity known as spontaneous tonsil hemorrhage, a highly rare complication of acute and/or chronic tonsillitis in our time, which used to have major severity and mortality rate in the pre-antibiotic era. Following next, we gather and analyze the information described in literature referred to this affliction, as well as the complementary tests required to define its fundamentally surgical management.
\end{abstract}

Key words: Spontaneous tonsillar hemorrhage, tonsillar hemorrhage, bilateral tonsillitis, spontaneous tonsillar bleeding, tonsillectomy.

\footnotetext{
Escuela de Medicina, Universidad del Desarrollo, Santiago, Chile.

Servicio Otorrinolaringología, Clínica Alemana, Santiago, Chile.

3 Departamento Otorrinolaringología, Facultad de Medicina, Universidad del Desarrollo, Santiago, Chile.

Los autores declaran no tener conflictos de interés.
}

Recibido el 20 de agosto de 2019. Aceptado el 11 de noviembre de 2019. 


\section{INTRODUCCIÓN}

Las patologías inflamatorias-infecciosas que afectan a las amígdalas palatinas son causa conocida de morbilidad tanto en la población pediátrica como adulta. La hemorragia amigdalina espontánea (HAE) es una complicación infrecuente de la amigdalitis aguda y/o crónica en la era antibiótica ${ }^{1}$. Hasta el año 2013 sólo 53 casos habían sido reportados en la literatura², los cuales se asociaban a infecciones bacterianas, mononucleosis infecciosa, sarampión, abscesos periamigdalinos, parafaríngeos 0 retrofaríngeos, malformaciones vasculares, aneurismas carotídeos, púrpura trombocitopénico idiopático ${ }^{3}$, enfermedad de von Willebrand $d^{4}$, hipertensión arterial severa ${ }^{5} \mathrm{y}$ cáncer de amígdalas, entre otros ${ }^{6-8}$. Griffies y cols la definieron como el sangrado continuo no iatrogénico de las amígdalas por una hora o la pérdida de más de $250 \mathrm{ml}$ de sangre independiente de la duración del sangrado $0^{6}$. Se ha reportado una incidencia mayor en pacientes jóvenes ${ }^{2}$, asociándose también a una tasa de mortalidad mayor. En la era preantibiótica estos cuadros eran graves, dado que en la mayoría de los casos se formaban abscesos cervicales profundos que provocaban erosión de grandes vasos, resultando en sangrados abundantes. Actualmente, la mayoría de las HAE son moderadas, como resultado del sangrado de vasos superficiales pequeños ${ }^{9}$. En este reporte

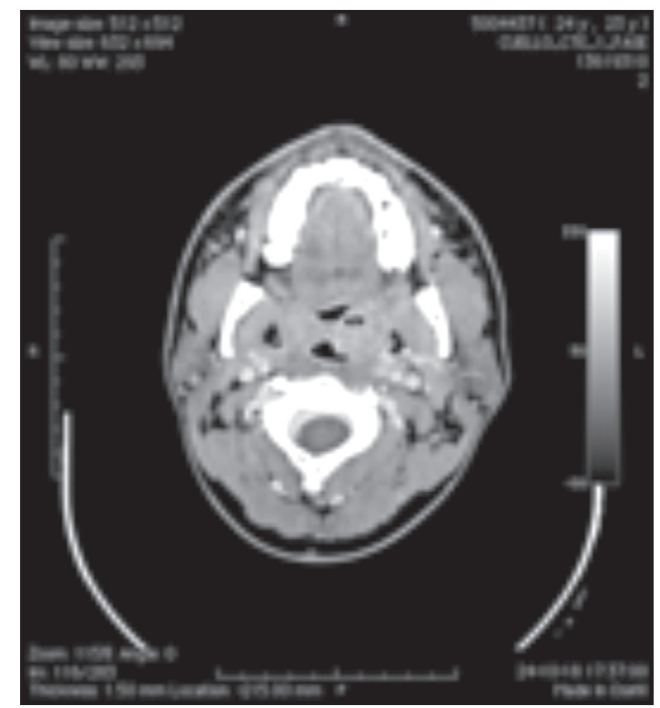

Figura 1. Tomografía computarizada de cuello con contraste, corte axial. Se evidencia aumento de volumen amigdalino bilateral mayor a izquierda, con presencia de burbujas de aire en amígdala izquierda. se describe un caso de amigdalitis hemorrágica espontánea abscedada, una entidad poco frecuente que requiere resolución quirúrgica precoz.

\section{CASO CLÍNICO}

Paciente masculino de 23 años de edad sin antecedentes mórbidos 0 quirúrgicos relevantes. Consulta en octubre del año 2018 refiriendo un cuadro clínico de 3 días de duración caracterizado por odinofagia intensa, disfagia y fiebre, a lo que se suma sangrado continuo por vía oral cuantificado aproximadamente en $30 \mathrm{ml}$, que inicia 4 horas previo al momento de la consulta. La anamnesis dirigida confirma la ausencia de historia previa de sangrado de similares características 0 amigdalitis aguda previa, antecedentes familiares de hemofilia 0 sangrado por otros sistemas. Ingresa al servicio de urgencias en buenas condiciones generales, hemodinámicamente estable.

El examen físico otorrinolaringológico constata un aumento de volumen cervical izquierdo y en polo superior de amígdala izquierda, con presencia de coágulo en su superficie, la cual se aprecia friable, con sangrado escaso. No se evidencia desviación de la úvula o trismus. Se solicita tomografía computarizada (TC) de cuello con contraste (Figuras 1 y 2), la que confirma aumento de volumen amig-

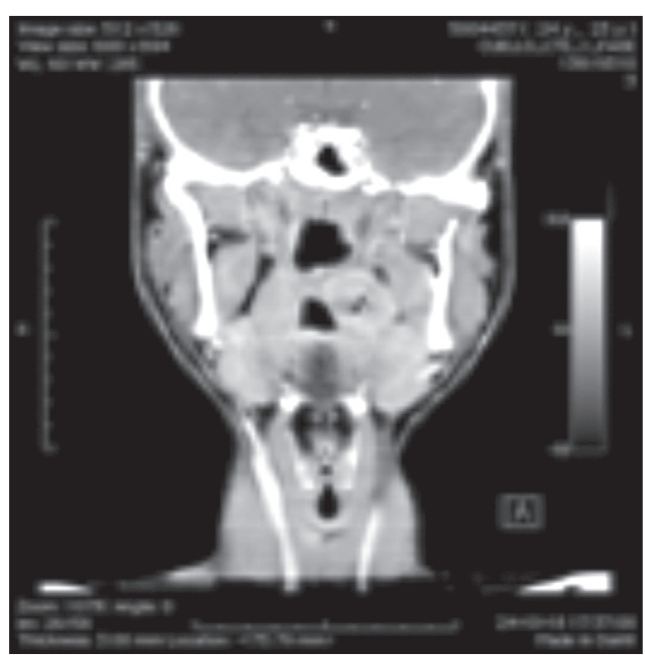

Figura 2. Tomografía computarizada de cuello con contraste, corte coronal. Se evidencia realce y edema difuso de componentes del piso de la boca, asimetría amigdalina con realce en anillo en amígdala izquierda con presencia de burbujas de gas en su interior. 
dalino bilateral mayor a izquierda, observándose además un área hipodensa con burbujas de gas en amígdala izquierda, la que presenta dimensiones de $17 \times 8 \times 16 \mathrm{~mm}$ en los diámetros transverso, anteroposterior y craneocaudal respectivamente; lo cual resulta concordante con un proceso inflamatorio-infeccioso de ambas amígdalas palatinas, con la presencia de un absceso periamigdalino izquierdo, asociado a adenopatías cervicales reactivas bilaterales. Se decide resolución quirúrgica, realizándose amigdalectomía bilateral, la cual resulta sin incidentes. El paciente evoluciona de manera favorable, sin episodios de sangrado durante el posoperatorio.

\section{DISCUSIÓN}

Si bien existen diversas indicaciones de amigdalectomía clásicamente conocidas, dentro de las cuales destacan amigdalitis recurrentes, hipertrofia amigdalina y abscesos amigdalinos a repetición, entre otras ${ }^{9-12}$; no debe pasarse por alto su indicación frente a la infrecuente pero importante entidad clínica definida como amigdalitis hemorrágica espontánea ${ }^{9,13-15}$ cuando ésta produce sangrados persistentes que no responden a medidas iniciales, siendo la amigdalectomía entonces, el procedimiento terapéutico fundamental.

En cuanto a la HAE en particular, la hemorragia amigdalina generalmente tiende a ser unilateral, más frecuentemente en el polo superior ${ }^{16,17}$. Si bien este paciente tenía un sangrado uni e ipsilateral al absceso periamigdalino que provenía del polo superior de la amígdala izquierda, el compromiso inflamatorio-infeccioso era bilateral, caso altamente infrecuente que no se había documentado en Chile hasta la fecha. Se realizó una búsqueda con el fin de encontrar un registro o caracterización de la incidencia de la HAE bajo diferentes palabras clave, sin encontrarse evidencia bibliográfica.

La fisiopatología detrás de este tipo de sangrados amigdalinos consiste en el aumento del flujo sanguíneo hacia las amígdalas como resultado de un proceso inflamatorio agudo local con subsecuente edema y congestión vascular, proceso durante el cual vasos sanguíneos superficiales y dilatados sufren necrosis y conducen a la hemorragia clínicamente evidente 0 referida por el pa- ciente $^{18}$. Esta entidad se presenta preferentemente en pacientes entre 20 y 30 años, sin predominio de género, encontrándose una duración promedio de los síntomas que varía entre 2 a 5 días previo a la consulta ${ }^{17,18}$, lo que coincide con el paciente presentado.

Si bien la HAE puede presentarse en contexto de cuadros como mononucleosis infecciosa, sarampión o incluso sífilis; la evidencia internacional disponible muestra que los casos que evolucionan a sangrado clínico evidente son muy infrecuentes ${ }^{17-19}$, algunos de los cuales pueden presentarse en estados de inmunosupresión importante ${ }^{20}$. Otros cuadros asociados, como los trastornos de la coagulación, entre los cuales se encuentra la enfermedad de von Willebrand constituyen una etiología aun menos frecuente que las antes mencionadas; sin embargo, se ha descrito el debut 0 diagnóstico de esta entidad en base a la HAE como primera manifestación; tal como ocurre además con el carcinoma amigdalino ${ }^{21}$. No obstante a lo anterior, la literatura es clave en recalcar que la amigdalitis recurrente es el principal factor de riesgo predisponente para la amigdalitis hemorrágica espontánea, seguida de los abscesos periamigdalinos y retrofaríngeos ${ }^{2,18}$. Este paciente no tenía historia previa de cuadros inflamatoriosinfecciosos previos tonsilares; infecciones como mononucleosis, sarampión o sífilis; 0 trastornos de la coagulación.

Para evaluar la extensión del compromiso infeccioso en tejidos circundantes o el tamaño de la colección abscedada ante una sospecha de un absceso periamigdalino, la TC de cuello con contraste representa un gran aporte, en la que puede evidenciarse realce y edema difuso de los componentes del suelo de la boca, aumento del tamaño tonsilar, asimetría del área periamigdalina por presencia de masa hipodensa con realce en anillo con o sin presencia de burbujas de gas en su interior, esto último secundario a la proliferación de microorganismos anaerobios ${ }^{22}$. Ante el diagnóstico de un absceso periamigdalino, en relación a la TC de cuello es posible tomar dos conductas; en ausencia de colección purulenta se continúa el tratamiento antibiótico de amplio espectro generalmente ya iniciado, en cambio, en presencia de dicha colección se drena el absceso en las siguientes 24 horas. Aquellos pacientes 
donde la TC demuestra la presencia de gas entre los tejidos cervicales tienen peor pronóstico que aquellos que no lo presentan; es por esto que la conducta quirúrgica inmediata tiene por objetivo vaciar el contenido purulento y debridar el tejido necrótico ${ }^{23}$.

Varios reportes en el pasado ya han descrito que frente a una $\mathrm{HAE}$, las primeras medidas siempre debiesen ir orientadas a lograr control del sangrado ${ }^{16,24}$-el cual la mayoría de las veces es superficial- ya sea a través de la aplicación de presión local, tórulas embebidas en agentes hemostáticos, nebulización con adrenalina 0 cauterización química local con nitrato de plata, entre otras medidas ${ }^{18,25-27}$. A la fecha, no se registra en la literatura en forma estandarizada qué hallazgos específicos en la TC constituyen indicación de estudio complementario con arteriografía, sin embargo, se sugiere su realización en caso de observarse engrosamiento mural concéntrico de los tejidos blandos circundantes a la arteria carótida 0 ante la presencia de una placa aterosclerótica no calcificada, ya que estos hallazgos pueden significar la presencia de un aneurisma 0 un hematoma mural a nivel de la arteria carótida interna ${ }^{28}$. También se sugiere realizar angiografía en aquellos casos que presenten sangrados severos -cuya cuantía no está estandarizadaen los que no se logra hemostasia local; donde además se recomienda amigdalectomía bilateral precoz ${ }^{2}$.

La resolución quirúrgica también es recomendada en aquellos casos en los que existe un flegmón 0 absceso amigdalino concomitante ${ }^{2}$. No obstante, ha quedado demostrado que, si bien las medidas de hemostasia local pueden ser útiles en la fase inicial del manejo, el control definitivo del sangrado debe ser de resorte quirúrgico, ya que los vasos que originan este sangrado requieren que la amígdala sea removida para ser accedidos ${ }^{29}$. Además, siempre debiese realizarse estudio anatomopatológico del tejido extraído, dada la posibili- dad de un carcinoma amigdalino ${ }^{2}$, aun cuando esto sea poco probable.

El diagnóstico clínico de hemorragias provenientes de vasos de mayor calibre frecuentemente se asocia a abscesos cervicales profundos que debilitan la pared de los vasos con tromboflebitis regionales y forman pseudoaneurismas que llevan a hemorragias fatales a posteriori ${ }^{1,30,31}$. La erosión carotídea suele presentarse con hemorragias centinela abruptas y recurrentes ${ }^{30,32}$-es decir, episodios de sangrados pequeños y repetitivos, premonitorios de una hemorragia masiva-además de sangrados retardados provenientes de pseudoaneurismas una vez resuelta la infección aguda. También se ha descrito la aparición de un síndrome de Horner ipsilateral y la parálisis inexplicable de los nervios craneales IX, X, XI y XII ${ }^{1,30}$, por lo que ante la presencia de estos signos debiese ser considerado realizar una arteriografía ${ }^{28,30,33,34}$.

\section{CONCLUSIÓN}

La HAE es una entidad clínica infrecuente y los cuadros abscedados lo son aún más. El compromiso amigdalino bilateral asociado a HAE es una entidad previamente no documentada en nuestro país. Si bien este cuadro representa un caso inusual dentro de las entidades otorrinolaringológicas, debe tenerse presente dado que su diagnóstico precoz y manejo oportuno disminuyen importantemente la morbimortalidad asociada. Aun cuando existen varias patologías relacionadas a la etiología de la $\mathrm{HAE}$, la más frecuentemente encontrada en la literatura internacional sigue siendo la amigdalitis recurrente, seguida de los abscesos periamigdalinos y retrofaríngeos. La TC de cuello con contraste representa una imagen complementaria de gran apoyo para definir conducta frente a una HAE en contexto de un absceso amigdalino. La resolución definitiva de este cuadro es quirúrgica y de manera precoz. 


\section{BIBLIOGRAFÍA}

1. Salinger S, Pearlman SJ. Haemorrhage from pharyngeal and peritonsillar abscess. Arch Otolaryngol 1933; 18: 464-509.

2. Salem A, Healy S, Pau H. Managment of spontaneous tonsillar bleeding: review. J Laryngol Otol 2010; 124: 470-3.

3. Levy S, Brodsky L, Stanievich J. Hemorrhagic tonsillitis. Laryngoscope 1989; 99; 15-8.

4. Lee DL, Soo G, van Hasselt CA. Spontaneous tonsillar hemorrhage due to von Willebrand's disease. J Laryngol Otol 2010; 124: 450-2.

5. Murty Ge, Samani NJ, Moloney JR. Bleeding tonsils. BMJ 1991; 302: 236.

6. GrifFIES WS, Wotowic PW, WILDES TO. Spontaneous tonsillar hemorrhage. Laryngoscope 1988; 98: 365-8.

7. John DG, Thomas PL, Semeraro D. Tonsillar Haemorrhage and measles. J Laryngol Otol 1988; 102: 64-6.

8. Kumra V, Vastola AP, Keiserman S, Lucente Fe. Spontaneous tonsillar hemorrhage. Otolaryngol Head Neck Surg 2001; 124: 51-2.

9. Darrow DH, Siemens C. Indications for tonsillectomy and adenoidectomy. Laryngoscope 2002; 112: 6-10.

10. Macaya A, Arruti I, Quer S. Amigdalectomía y Adenoidectomía: indicaciones, técnicas y complicaciones. SEORL, libro virtual, sección IV: cavidad oral, faringe, esófago, capítulo 79, págs. 1-15.

11. Cervera J, Del Castillo M, Gómez Ja, Gras JR, Pérez B, Villafruela MA. Indicaciones de Adenoidectomía y Amigdalectomía: Documento de Consenso entre la Sociedad Española de Otorrinolaringología y Patología Cervicofacial (SEORL) y la Asociación Española de Pediatría (AEPED). Acta Otorrinolaringol Esp 2006; 57: 59-65.

12. Aedo C, Muñoz D. Indicaciones no tradicionales de amigdalectomía. Rev Otorrinolaringol Cir Cabeza Cuello 2010; 70: 165-74.

13. McCormick MS, Hassett P. Spontaneous haemorrhage from the tonsil (a case report). $J$ Laryngol Otol 1987; 101: 613-6.

14. Darrows D. Dilemas en el abordaje de la amígdala. Manual de Otorrinolaringología IAPO, FALTA AÑO; 81-7.
15. Walbaum B, Winter M, Huidobro B. Amigdalitis hemorrágica: presentación de un caso. Rev Otorrinolaringol Cir Cabeza Cuello 2016; 76 : 82-5.

16. Rocha SCM, DelL’Aringa AR, Nardi JC, Kobari K, DE MeLo C. Spontaneous tonsillar hemorrhage. Rev Bras Otorrinolaringol 2007; 73: 287.

17. Ríos OAB, Lessa RM, Grwnato L. Hemorragia Espontânea da Amígdala. Relato de Caso e Revisão da Literatura. Rev Bras Otorrinolaringol 1999; 65: 457-60.

18. Lourenço EA, Almeida CIR, Pinto CAL, Silva Júnior WVS. Hemorragia Espontânea de Amígdalas Palatinas. Relato de Dois Casos. Rev Bras de Otorrinolaringol 1991; 57: 36-9.

19. Koay CB, Norval F. An Unusual Presentation of an Unusual Complication of Infectious Mononucleosis: Haematemesis and Melena. $J$ Laryngol Otol 1995; 109: 335-6.

20. John DG, Thomas PL, Semerano D. Tonsillar haemorrhage and measles. J Laryngol Oto/1999; 102: 64-6.

21. GUMPRECHT TF, CICHOR JV. Otolaryngology and von Willebrands Disease. Arch Otolaryngol 1981; 107: 491-3.

22. Assing O, Ballesta M, Pamies J, Vigrer RM, Palacios P, Arango JJ. Patologia Infecciosa de la Cavidad oral y el Cuello. (2012) $31^{\circ}$ Congreso Sociedad Española Radiología Médica (SERAM). Granada, España.

23. Seguí P, Jiménez M, Pérez CA. Complicaciones de las infecciones orales y faríngeas. SEORL, libro virtual, sección III: cavidad oral y farínge, capítulo 78, págs. 1-18.

24. Yumha MJ, Boettiger P, Arias R. Adenoamigdalitis hemorrágica espontánea, revisión de la literatura y presentación de tres casos. Rev Otorrinolaringol Cir Cabeza Cuello 2016; 76: 77-81.

25. Rowlands RG, Hickin L, Hinton AE. Novel Use of a Nebulised Adrenaline in the Treatment of Secondary Oropharyngology Haemorrhage. $J$ Laryngol Otol 2002; 116: 123-4.

26. Kım YS, Hong SJ, Lee SH, Kwon SY, CHoI $\mathrm{JH}$. Spontaneous tonsillar hemorrhage and post-tonsillectomy hemorrhage. Clin Exp Otorhinolaryngol 2010; 3: 56-8.

27. Shatz A. Spontaneous tonsillar bleeding; secondary to acute tonsillitis in children. Int $J$ Otorhinolaryngol 1993; 26: 181-4. 
28. Kasravi N, Leung A, Silver I, Burneo JG. Dissection of the internal carotid artery causing Horner syndrome and palsy of cranial nerve XII. Can Med Assoc J 2010; 182: E373-7.

29. Vlastarakos PV, Iacovou E. Spontaneous tonsillar hemorrhage managed with emergency tonsillectomy in a 21-year-old man: a case report. J Med Case Rep 2013; 7: 192. doi:10.1186/17521947-7-192.

30. Eneroth CM, Tham R. Pseudoaneurysm of the internal carotid artery. A warning of septic erosion. Acta Otolaryngol 1971; 72: 445-50.

31. Blum DJ, McCaffrey TV. Septic Necrosis of the Internal Carotid Artery: A Complication of
Peritonsillar Abscess. Otolaryngol Head Neck Surg 1983, 91: 114-8.

32. COHEN J, RAD I. Contemporary management of carotid blowout. Curr Opin Otolaryngol Head Neck Surg 2004; 12: 110-5.

33. Mangat SS, Nayak H, Chandna A. Horner's Syndrome and sixth nerve paresis secondary to a petrous internal carotid artery aneurysm. Semin Ophthalmol 2011; 26: 23-4.

34. Fabiano L, Bernardi D, Carvalho A, Celso Wa, Ferreira F, Morita ME, Mauad W, et al. Hypoglossal nerve palsy as the sole manifestation of spontaneous internal carotid artery dissection. Arq Neuropsiquiatr 2009; 67: 107-8. 